\title{
Free gait in a shallow pool accelerates recovery after exercise in model mice with fibromyalgia
}

\author{
Taiki Nakata ${ }^{1,2,4}$, Atsushi Doi ${ }^{2,3, * * *}$, Daisuke Uta ${ }^{4}$, Min-Chul Shin ${ }^{2,3}$, Megumu Yoshimura $^{5}$ \\ 'Department of Rehabilitation, Kumamoto-Saiseikai Hospital, Kumamoto, Japan \\ ${ }^{2}$ Graduate School of Health Science, Kumamoto Health Science University, Kumamoto, Japan \\ ${ }^{3}$ Department of Rehabilitation, Kumamoto Health Science University, Kumamoto, Japan \\ ${ }^{4}$ Department of Applied Pharmacology, Faculty of Pharmaceutical Sciences, University of Toyama, Toyama, Japan \\ ${ }^{5}$ Department of Orthopedic Surgery, Nakamura Hospital, Fukuoka, Japan
}

This study aimed to determine the effect of pool gait exercise using fibromyalgia-induced model mice. The sensory threshold, locomotive behavior, electrocardiogram, and onset time after the gait test in shallow water using male $\mathrm{C} 57 \mathrm{BL} / 6 \mathrm{~J}$ mice (weight, 30-35 g; $\mathrm{n}=21$ ) were investigated. To induce fibromyalgia in model mice, reserpine was injected intraperitoneally into wild-type mice once a day for 3 days. Subsequently, the fibromyalgia-induced model mice were randomly classified into two groups as follows: the control group $(n=11)$ and the pool gait group $(n=10)$. The mice in the pool gait group walked in the same cage containing shallow warm water 5 times per week. Both groups underwent sensory thresholds and video recordings to determine locomotive behaviors weekly. Further, both heart rate and video recordings for ob- servation of a recovery after the gait test in shallow water were undertaken (control group; $n=5$, pool gait group; $n=5$ ). The pool gait did not affect sensory thresholds and locomotive behavior; however, in the pool gait group, both the recovery after the test, such as onset time and gait distance, were considerably better than those of the control group. Furthermore, changes in heart rate and heart rate irregularity after the test were more apparent in the control group than in the pool gait group. The free gait in a shallow pool accelerated recovery after exercise, unlike the sensory threshold.

Keywords: Shallow pool gait, Behavior analysis, Fibromyalgia-induced model mouse

\section{INTRODUCTION}

Fibromyalgia (FM) is a disease that causes widespread musculoskeletal pain, which affects a patient's physical and psychological conditions causing fatigue, and sleep and mood disorders (Bazzichi et al., 2020). Recently, FM patients have been found to have impaired cardiac function (da Cunha Ribeiro et al., 2011) and to experience a high risk of coronary heart disease-related events (Tsai et al., 2015).

Therapeutic exercise has been performed for such FM patients (Andrade et al., 2017). Therapeutic exercise potentially stimulates the central nervous system (Maejima et al., 2019) and muscular contraction (as part of the peripheral nervous system) (Vlietstra et al., 2018), as well as cardiovascular (Vlietstra et al., 2018), and autonomic systems (Pearson and Smart, 2018). The exercise regimen contains either active or resistive exercises. The active exercises may not so useful for muscle strength. On the other hand, although resistive exercises are a better way to develop muscle strength compared to active exercises, during the exercise, health professionals must always be mindful of resistive training-induced pain enhancement in FM patients. These exercises are generally performed either on the ground or in water. Water-walking has often been utilized as a therapeutic exercise for painful arthritic conditions such as osteoarthritis (Dias et al., 2017) and rheumatoid arthritis (Bilberg et al., 2005). It has been reported that exercise in chest-high warm water decreases pain and improves cogni-

\footnotetext{
*Corresponding author: Atsushi Doi iD https://orcid.org/0000-0001-8913-0124 Graduate School of Health Science, Kumamoto Health Science University,

Kumamoto 861-5598, Japan

E-mail: atsushidoi01@gmail.com

\#These authors contributed equally to this study as co-first authors.

Received: September 2, 2020 / Accepted: September 20, 2020
}

This is an Open Access article distributed under the terms of the Creative Commons Attribution Non-Commercial License (https://creativecommons.org/licenses/by-nc/4.0/) which permits unrestricted non-commercial use, distribution, and reproduction in any medium, provided the original work is properly cited. 
tive function in patients with FM (Mannerkorpi et al., 2009; Segura-Jiménez et al., 2013). One the other hand, previous studies have reported that aquatic exercise only slightly improved pain (Tomas-Carus et al., 2008), or the training did not statistically improve pain compared to balneotherapy (Altan et al., 2004). Thus, the effectiveness of aquatic exercises in alleviating pain requires further research in the context of FM patients.

Therefore, this study aimed to investigate the effect of gait exercise in shallow water on pain, cardiac function, and locomotive behavior including motor recovery after exercise using FM-induced model mice.

\section{MATERIALS AND METHODS}

\section{Animals}

Male C57BL/6J mice (weight, 30-35 g; $\mathrm{n}=21$ ) were purchased from Kyudo, Inc. (Kumamoto, Japan) and housed under controlled temperature $\left(24^{\circ} \mathrm{C} \pm 1^{\circ} \mathrm{C}\right)$ and humidity $(55 \% \pm 10 \%)$, with a 12 hour light-dark cycle, and with food and water freely available. The animal experiments were approved by the Animal Care Committees of Kumamoto Health Science University (approval number: 19-03), and were conducted in accordance with the National Institute of Health Guide for the Care and Use of Laboratory Animals (NIH publications No. 80-23, revised 1996).

\section{Reserpine-injected FM model mice}

To induce FM in the model mice, reserpine was injected through the intraperitoneal route $(1 \mathrm{~mL} / \mathrm{kg})$ into wild-type mice once a day for 3 days (Nagakura et al., 2009). Reserpine, purchased from Nacalai Tesque (product No.: 30013-81, Kyoto, Japan), was dissolved in $100 \%$ glacial acetic acid $(1 \mathrm{mg} / 0.05 \mathrm{~mL}$ ) (solution A). Subsequently, distilled water $(1 \mathrm{mg} / 0.95 \mathrm{~mL})$ was added to solution A (solution B, $1 \mathrm{mg} / 1 \mathrm{~mL}$ ), containing $5 \%$ glacial acetic acid to create solution $\mathrm{B}$. Following this, solution $\mathrm{B}$ was diluted to a final concentration of $0.5 \%$ acetic acid with distilled water (stock solution), which was subcutaneously injected into the mice 3 times, at a dosage of $1 \mathrm{~mL} / \mathrm{kg}$.

\section{Two kinds of therapeutic gait exercises for the FM-induced model mice}

After the third reserpine injection, the mice were randomly classified into two groups as follows: the control group $(\mathrm{n}=11)$ and the pool exercise group $(n=10)$ (Fig. 1A). The mice in the control group could walk freely in the rat cage (Fig. 1B). On the contrary, mice in the pool gait group walked in a similar cage containing shallow warm water (Fig. 1B). Different interventions were conducted on the mice in these two groups over a 4-week duration ( 5 times/wk). Similarity was observed in the walking duration between both groups (Fig. 1B). The temperature of the water in the cage ranged between $40^{\circ} \mathrm{C}-42^{\circ} \mathrm{C}$, and the level of the shallow warm water in the cage ranged between $1.0-1.5 \mathrm{~cm}$ to prevent the mice from drowning.

\section{Measurement of the sensory threshold with electrical-and} heat-stimulation

Sensory thresholds were measured using 5-, 250-, and 2,000-Hz sine electrostimulation (product: STG-4002-160 $\mu \mathrm{A}$, Multichannel Systems Inc., Reutlingen, Germany) before and after the reserpine injections (Fig. 1C, evaluation A) (Doi et al., 2018). The three different electrostimulation frequencies used in this study stimulated $A \beta$ fibers $(2,000 \mathrm{~Hz}), A \delta$ fibers $(250 \mathrm{~Hz})$, and $C$ fibers $(5 \mathrm{~Hz})$. An awake mouse was immobilized in a plastic tube, which was clamped using an adjustable magnetic base and stand (product No.: A-2, Shinwa Rules Co., Sanjyo, Niigata, Japan) and a laboratory clamp (product No.: NC-3, Kenis, Osaka, Japan). Ball-type bipolar electrodes (product No.: EKL2-2020, Bio-Research Center, Nagoya, Japan) were placed on the plantar surface of the right hind foot, and electrostimulation was applied to the plantar surface as the knee joint was maximally flexed. Further, the ankle joint was dorsiflexed, with the mouse in a supine position. The electrostimulation-induced withdrawal reflex of the mouse's hind limb resulted in a loss of contact with the electrode. The time from the onset of electrostimulation to the appearance of the withdrawal reflex was measured, and the intensity at which the withdrawal reflex occurred was calculated $(\mu \mathrm{A})$ (Doi et al., 2018).

Another sensory threshold was measured using heat-stimulation before and after the reserpine injection (Fig. 1C, evaluation A). An awake mouse was immobilized in a plastic tube, which was clamped using an adjustable magnetic base and stand (product No.: A-2, Shinwa Rules Co., Sanjyo, Niigata, Japan) and a laboratory clamp (product No.: NC-3, Kenis, Osaka, Japan). In the measurement of the sensory threshold, a probe with a $25-\times 25-\mathrm{mm}$ surface was placed on the plantar surface of the right hind foot, and heat stimuli (product No.: Intercross-210, Intercross Inc., Tokyo, Japan) was applied to the plantar surface, with the mouse in a prone position. The Intercross-210 was connected to a personalized computer (PC), and the temperature was displayed on the PC screen. The time from the onset of heat-stimulation to the observation of a withdrawal reflex was recorded. 


\section{Weekly evaluation of locomotive behavior during the gait} exercises

Weekly video recordings of the free gait for each FM-induced model mouse was undertaken in a cage following the completion of the course of reserpine injections (Fig. 1C, evaluation B) (Fig. 1B, control group; $n=11$, pool gait group; $n=10$ ). The recordings were performed using the video function of a digital camera (product No.: TZ-35, Casio, Tokyo, Japan) for > 60 sec. Tracking, walking direction, walking distance $(\mathrm{cm} / 40 \mathrm{sec})$, maximum speed $(\mathrm{cm} / \mathrm{sec})$, and average speed $(\mathrm{cm} / \mathrm{sec})$ were the main parameters for the analysis. Subsequently, a video camera on top of the cage recorded the movements of each mouse (Doi et al., 2017). In the analysis of the gait videos, Avidemux (http://fixounet.free.fr/avidemux/), Any Video Converter (http://www.any-video-converter.com/products/ for_video_free/), VirtualDub (http://www.virtualdub.org/), and ImageJ (https://fiji.sc/), which are open-source software programs, were used. The videos were cut with Avidemux and edited with

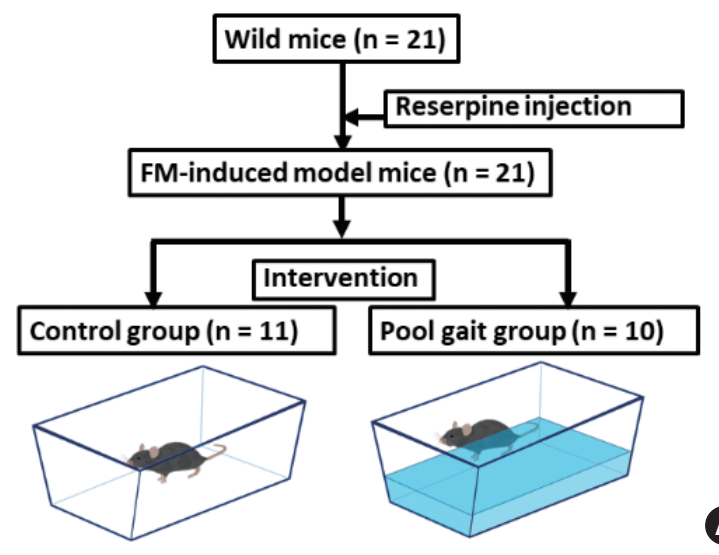

Any Video Converter and VirtualDub. The edited videos were then analyzed with ImageJ.

\section{Behavior evaluation during and after the gait exercise in the shallow water}

We evaluated the locomotive behavior during and after the gait exercise in shallow water for both groups (Fig. 1C, evaluation C and $D$, control group; $n=5$, pool gait group; $n=5$ ). In this evaluation, the video recordings were undertaken during and after the 5 -min gait exercise in the water at 4 weeks after the reserpine injection for both the groups. Following the completion of the 5-min gait exercise, both onset time of movement and movement were equally evaluated after the gait exercise. Both the way and analysis of the recordings were same as that in the "Weekly evaluation of locomotive behavior."

\section{The protocol of the intervention}

Control group (free gait without shallow warm water)

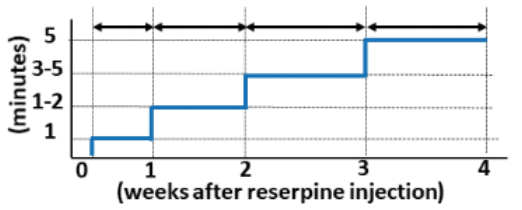

Pool gait group (free gait with shallow warm water)

A

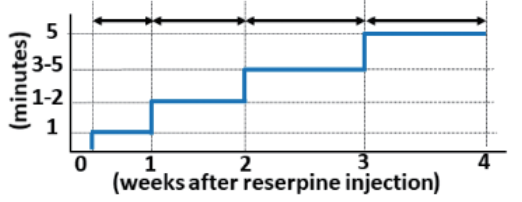

B

\section{Experimental protocol}

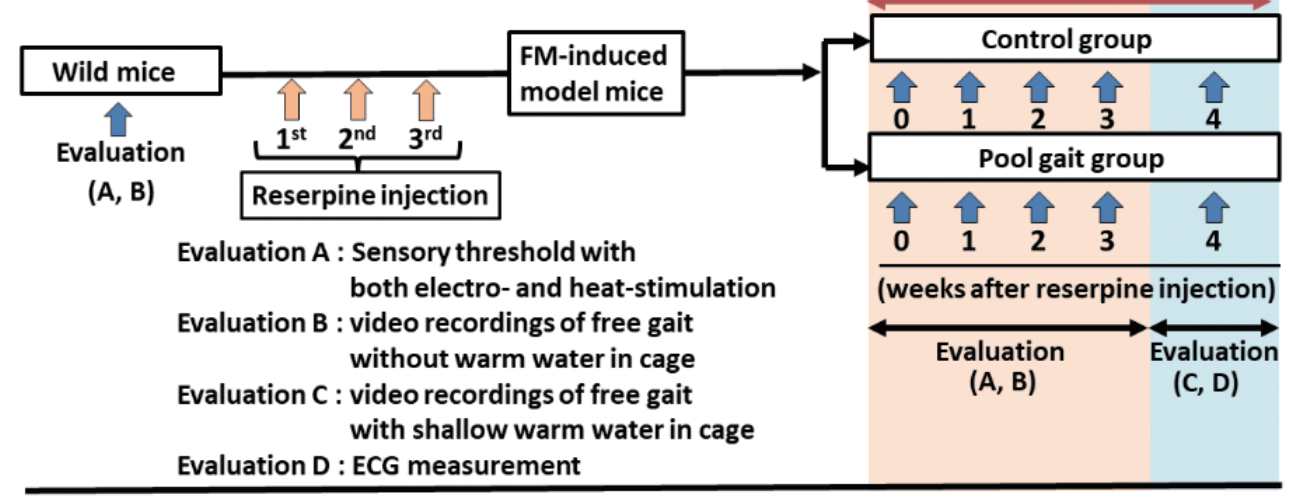

Fig. 1. A flow chart of the experimental group, intervention, and experimental protocol. (A) A flow chart of the group classification for the experiment. (B) The intervention protocol for the control and pool gait groups. (C) The experimental protocol for fibromyalgia (FM)-induced model mice. ECG, electrocardiogram. 


\section{Measurement of electrocardiogram before and after the gait exercise in shallow water}

We recorded the electrocardiogram (ECG) results during and after the gait exercise in shallow water at 4 weeks after the reserpine injection for both groups. In relation to the ECG recordings, with the mice placed in a prone position, disposable, self-adhesive $\mathrm{Ag} / \mathrm{AgCl}$ snap dual electrodes (product No.: \#272S, Noraxon, Scottstale, AZ, USA) were split into two parts and used to measure the ECG of the mice (Fig. 1C, evaluation $C$ and D). One part

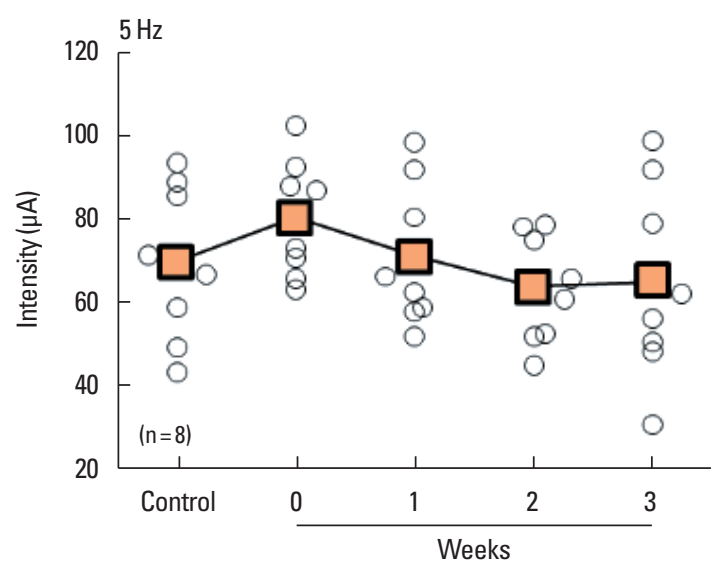

(1)
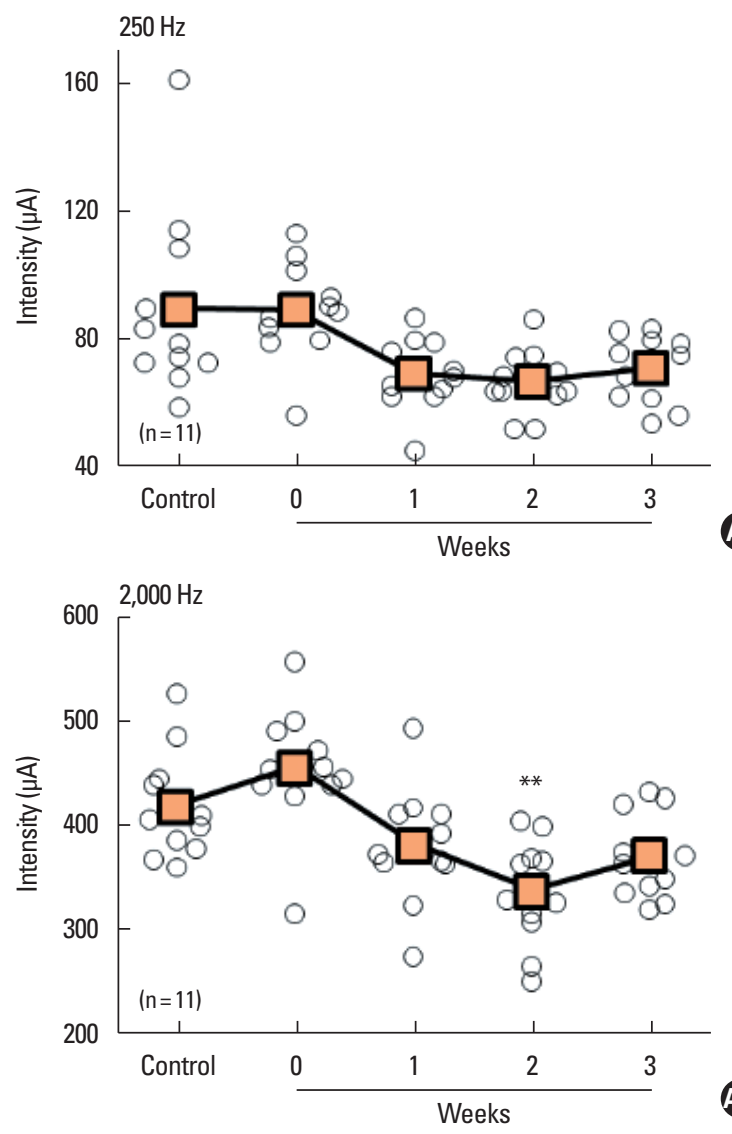

Aii

Aii) of the electrode was positioned on the palmar surface of the right forefoot as a positive electrode, and the other part of the electrode was placed on the plantar surface of the left hind foot as a negative electrode. The ECG was recorded before and after the gait exercise in the shallow water. The recorded ECG signals were amplified using a differential amplifier (product: model 1700, AM-System, Sequim, WA, USA) and were digitized using an Axon Digidata 1322 digitizer (product: Dagidata1322A, Molecular Device, San Jose, CA, USA). Heart rate and heart rate irregularity scores were
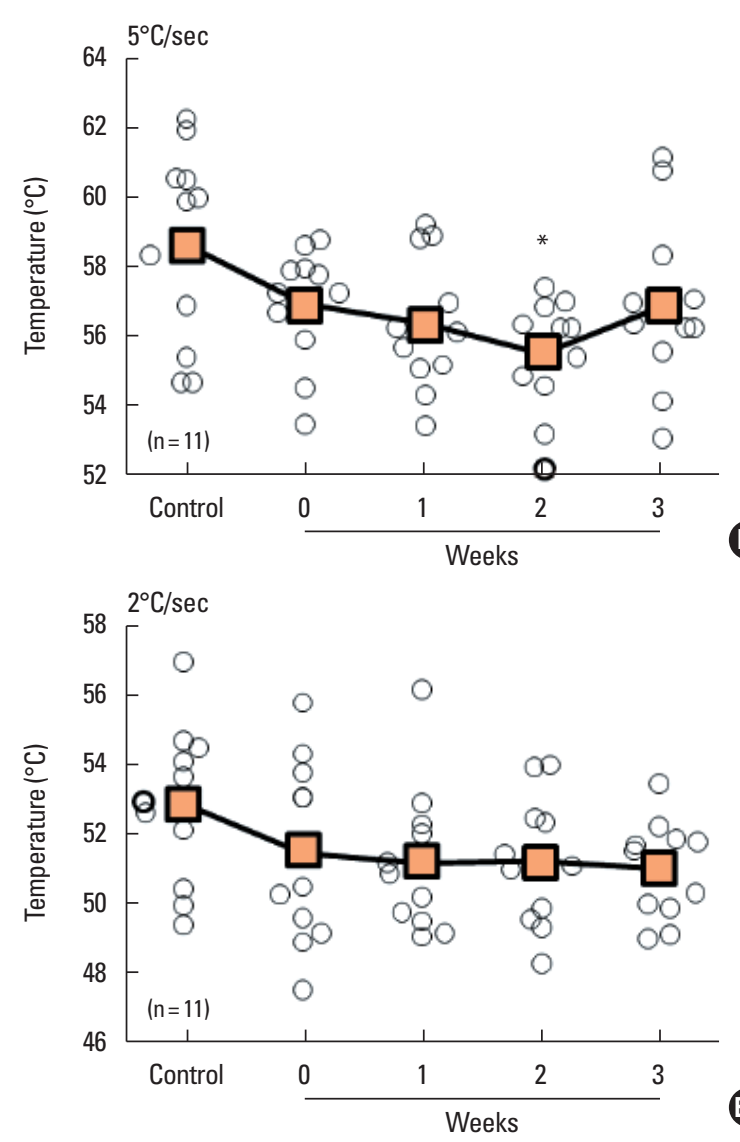

Fig. 2. Electrostimulation- and heat-evoked sensory threshold in the control group. A sensory threshold using a $5-\mathrm{Hz}$ sine wave (Ai), a 250- $\mathrm{Hz}$ sine wave (Aii), and a $2,000-\mathrm{Hz}$ sine wave (Aiii, ${ }^{* *} P<0.01$ ). A sine wave is an electrical stimulation used to determine the sensory threshold. The sensory threshold with heat stimulus $\left(5^{\circ} / \mathrm{sec}\right)\left(\mathrm{Bi},{ }^{*} P<0.05\right)$ and heat stimulus $(2 \%$ sec) (Bii). 
consequently analyzed using DataView 11 (software, University of St Andrew, UK, https://www.st-andrews.ac.uk/ wjh/dataview/). The heart rate irregularity score was estimated for each cycle through the application of the formula below (for consecutive cycle length values): $\mathrm{Sn}=100 *$ ABS $(\mathrm{Pn}-\mathrm{Pn}-1) / \mathrm{Pn}-1,(\mathrm{Sn}=$ score of the $\mathrm{nth}$ cycle, $\mathrm{Pn}=$ period of the nth cycle, $\mathrm{Pn}-1=$ period of the cycle preceding the nth cycle, $\mathrm{ABS}=$ the absolute value) (Barthe and $\mathrm{Cl}-$ arac, 1997).

\section{Statistical analysis}

Experimental data were expressed as mean \pm standard deviation. Single comparisons were conducted using the Mann-Whitney $U$-test and Wilcoxon signed-rank test for unpaired and paired groups, respectively. Further, the Friedman test with Bonferroni correction was used for more than three groups. Moreover, both repeated measures analysis of variance (ANOVA) and the MannWhitney $U$-test with the Bonferroni correction as a post hoc test were also utilized. Values of $P<0.05$ were considered statistically significant. All statistical analyses were performed using EZR
(Saitama Medical Center, Jichi Medical University, Saitama, Japan), which is a graphical user interface for $\mathrm{R}$ (The $\mathrm{R}$ Foundation for Statistical Computing, Vienna, Austria). More precisely, it is a modified version of the $\mathrm{R}$ commander designed to add statistical functions that are frequently used in biostatistics (Kanda, 2013).

\section{RESULTS}

\section{Modulation of sensory thresholds and locomotive behavior in FM-induced model mice}

The sensory thresholds and locomotive behavior of the FM-induced model mice were examined. A decrease was observed in the sensory thresholds $(\mu A)$ with electrical stimulation at frequencies of $5 \mathrm{~Hz}$ (Fig. 2Ai), $250 \mathrm{~Hz}$ (Fig. 2Aii), and 2,000 Hz (Fig. 2Aiii); however, no statistically significant change was observed, except for electrical stimulation at a frequency of $2,000 \mathrm{~Hz}\left({ }^{* *} P=0.0024\right)$ (Fig. 2Aiii). On the contrary, the sensory thresholds $\left({ }^{\circ} \mathrm{C}\right)$ using heat stimuli significantly decreased at $5 \% \sec \left({ }^{*} \mathrm{P}=0.019\right)(\mathrm{Fig} .2 \mathrm{Bi})$, but not at $2 \%$ sec (Fig. 2Bii).

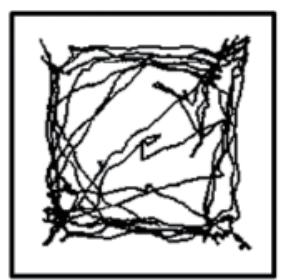

Control

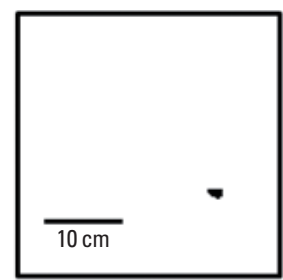

oWeek

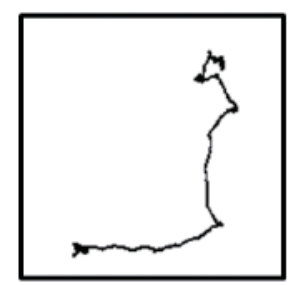

1 Week

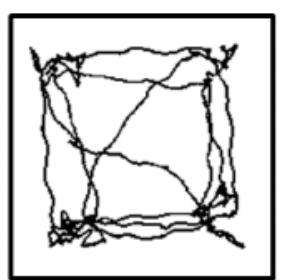

2Weeks

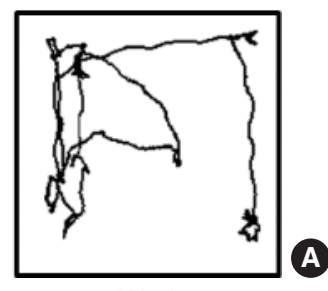

3Weeks
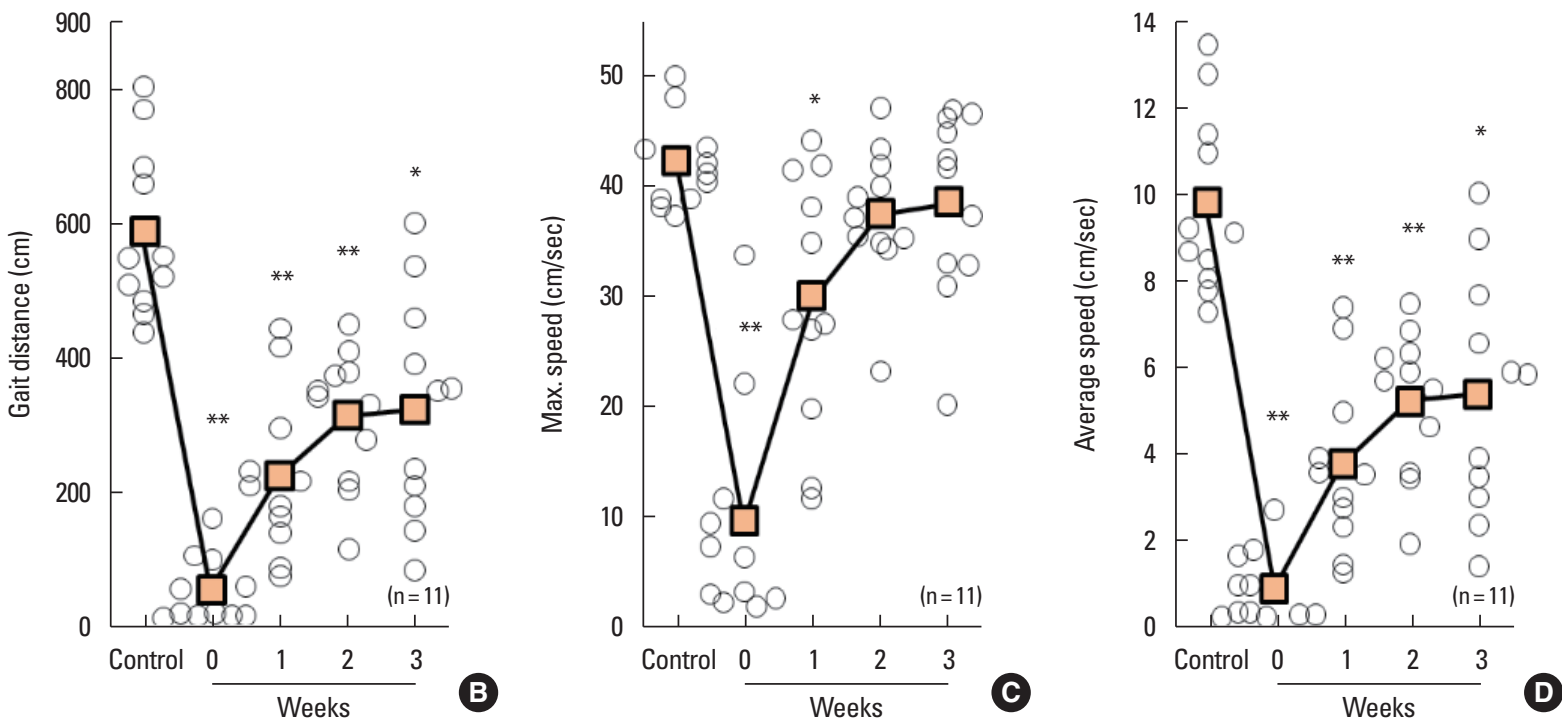

Fig. 3. Weekly changes in locomotive behavior in the control group. (A) An example of gait tracking in the FM-induced model mice. Changing the gait distance (B; $\left.{ }^{*} P<0.05,{ }^{* *} P<0.01\right)$, maximum speed $\left(C ;{ }^{*} P<0.05,{ }^{*} P<0.01\right)$, and average speed $\left(\mathrm{D} ;{ }^{*} P<0.05,{ }^{*} P<0.01\right)$ in the control group. 


\section{Change of locomotive behavior in FM model mice}

The locomotive behavior of the mice that were evaluated using parameters such as gait distance $(\mathrm{cm})$, maximum speed $(\mathrm{cm} / \mathrm{sec})$, and average speed $(\mathrm{cm} / \mathrm{sec})$, was suppressed due to the reserpine injection ( $\left.{ }^{*} P=0.0293,{ }^{* *} P=0.0098\right)$ (Fig. 3A-D). In addition, gradual recovery in both movement and speed were observed (Fig. 3A-D).

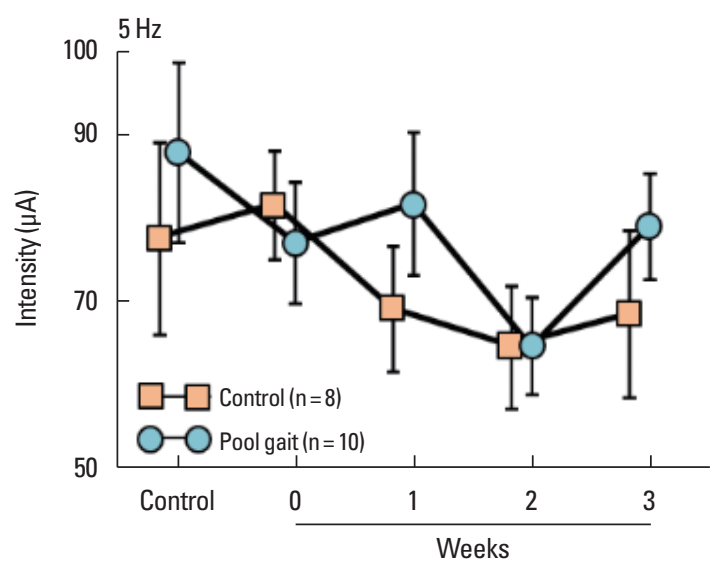

(1)
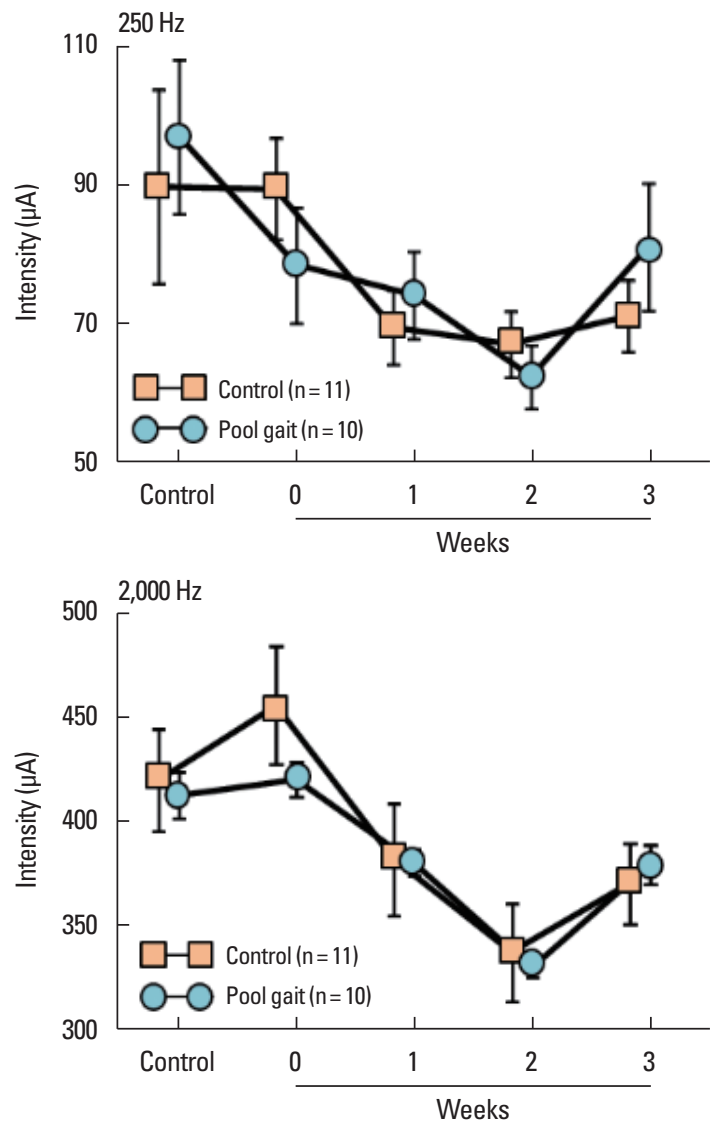

\section{Comparison of sensory thresholds with or without free} gait in shallow warm water

The control $(\mathrm{n}=8-11)$ and pool gait groups $(\mathrm{n}=10)$ were compared in the evaluation of sensory thresholds $(\mu \mathrm{A})$ with three kinds of electro-stimulus frequencies. However, no statistically significant differences were observed between the groups (Fig. 4Ai, 4Aii, and 4Aiii) Furthermore, in relation to the sensory threshold with heat-stimulus, no statistically significant differences were observed between the groups (Fig. 4Bi and 4Bii).
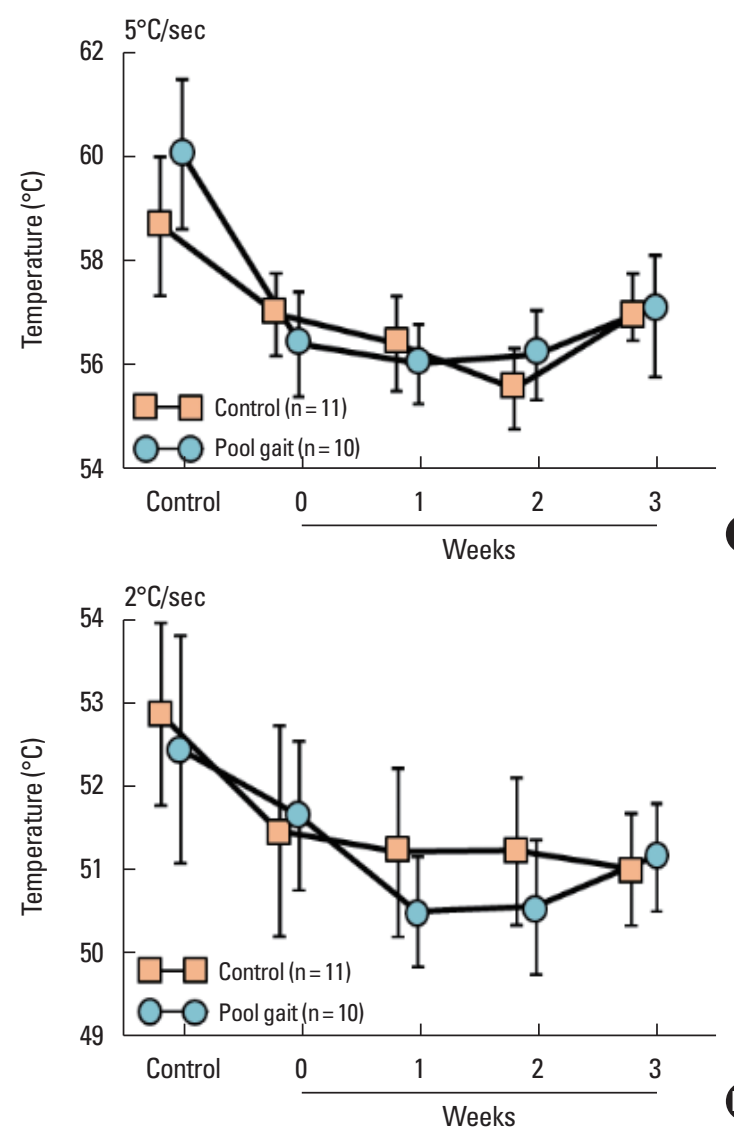

Fig. 4. Weekly changes in the sensory threshold in the control and pool gait groups. (Ai) A comparison of the sensory threshold with a $5-\mathrm{Hz}$ sine wave between the control and the pool gait groups. (Aii) A comparison of the sensory threshold with a $250-\mathrm{Hz}$ sine wave between the control and the pool gait groups. (Aiii) A comparison of the sensory threshold with a 2,000-Hz sine wave between the control and the pool gait groups. (Bi) A comparison of the sensory threshold with heat stimulus between the control and the pool gait groups ( $5 \%$ sec). (Bii) A comparison of the sensory threshold with heat stimulus between the control and the pool gait groups $(2 \%$ sec). 
Comparison of locomotive behavior with or without free gait in shallow warm water

The locomotive behavior of the mice in the two groups was com-

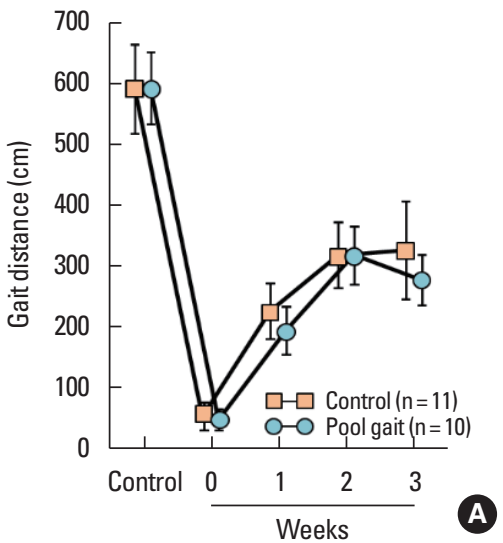

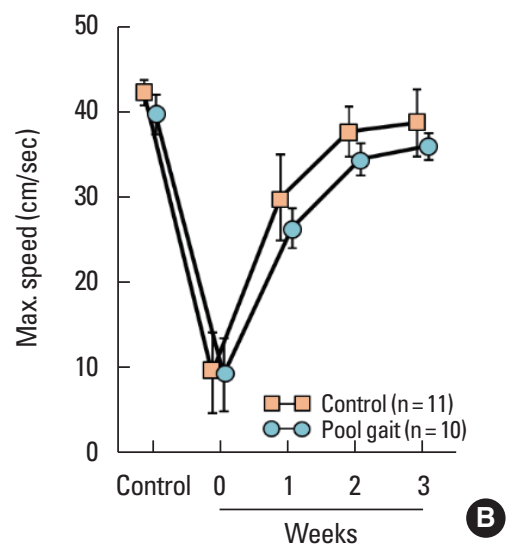

pared. However, no statistically significant differences were observed in gait distance (Fig. 5A-C).

Fig. 5. Weekly changes in locomotive behavior in the control and pool gait groups. Changing the gait distance (A), maximum speed (B), and average speed (C) for the control and the pool gait groups.
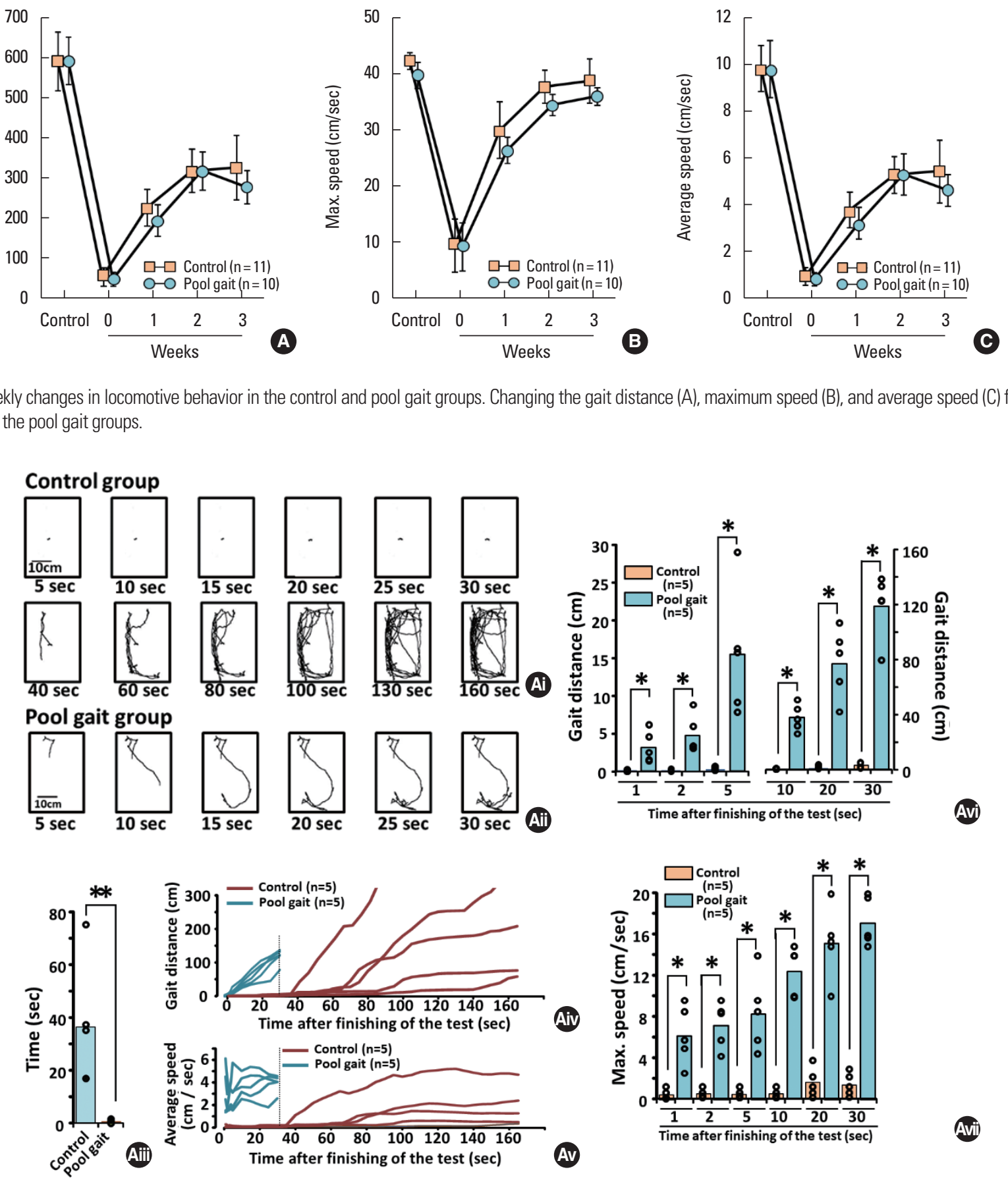

Fig. 6. Comparison of the motor recovery in the control and pool gait groups after exercise in shallow water. (Ai and Aii) An example of gait tracking in the control and pool gait groups after the exercise in shallow water. (Aiii) The difference in time of onset of movement after the exercise between the control and the pool gait groups $\left.{ }^{* *} P<0.01\right)$. (Aiv) The time course of the gait distance following the completion of the exercise. (Av) The time course of the average speed following the completion of the exercise. (Avi) The gait distance at time $(1,2,5,10,20$, and $30 \mathrm{sec})$ following the completion of the exercise $\left({ }^{*} P<0.05\right)$. (Avii) The maximum speed at the point of the time $(1,2,5,10,20$, and $30 \mathrm{sec})$ following the completion of the exercise $\left.{ }^{*} P<0.05\right)$. 


\section{Comparison of motor recovery with or without free gait in shallow warm water}

The mice's behavior concerning movement after the gait exercises in the warm water was compared between the groups (Fig. 6). The mice in the control group remained stationary at the 30-sec time point following the free gait in shallow warm water (Fig. 6Ai); however, mice in the pool group started moving quickly following the completion of the free gait in shallow warm water (Fig. 6Aii). The onset time of movement after the free gait in shallow warm water was significantly shorter in the pool group than in the control group $\left({ }^{* *} P=0.00794\right)$ (Fig. 6Aiii). Further, gait distance and maximum speed were significantly greater in the pool group than

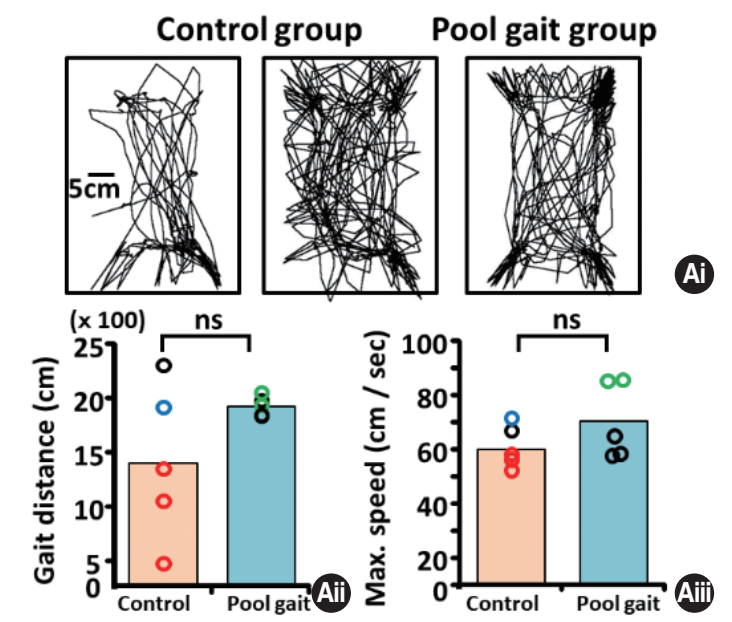

Fig. 7. Comparison of the cardiac function in the control and pool gait groups after exercise in shallow water. (Ai) Three examples of gait tracking during the exercise in shallow water. (Aii) Gait distance between the control and the pool gait groups during the exercise (ns). (Aiii) The maximum speed of the control and the pool gait groups during the exercise (ns). (Bi) An example of electrocardiogram (ECG) signals in the control group before and after the exercise. (Bii) A second example of ECG signals in the pool gait group before and after the exercise. (Biii) A third example of ECG signals in the pool gait group before and after the exercise. (Biv) The change in heart rate before and after the exercise in the control and pool gait groups. (Bv) Changes in the heart rate irregularity score before and after the exercise in the control and pool gait groups. ns, not significant difference. in the control group (gait distance; ${ }^{*} P<0.00001$ with repeated measures ANOVA and ${ }^{*} P=0.04764$ with Mann-Whitney $U$-test, Fig. 6Aiv and 6Avi, maximum speed; ${ }^{*} P<0.00001$ with repeated measures ANOVA and ${ }^{*} P=0.04764$ with Mann-Whitney $U$-test) (Fig. 6Av and 6Avii).

\section{Comparison of the cardiac function with or without the free gait in the water}

We compared the gait distance and maximum speed during the free gait in warm water between the two groups (Fig. 7Ai). No statistically significant difference in the average was observed between the two groups with regard to gait distance and maxi-
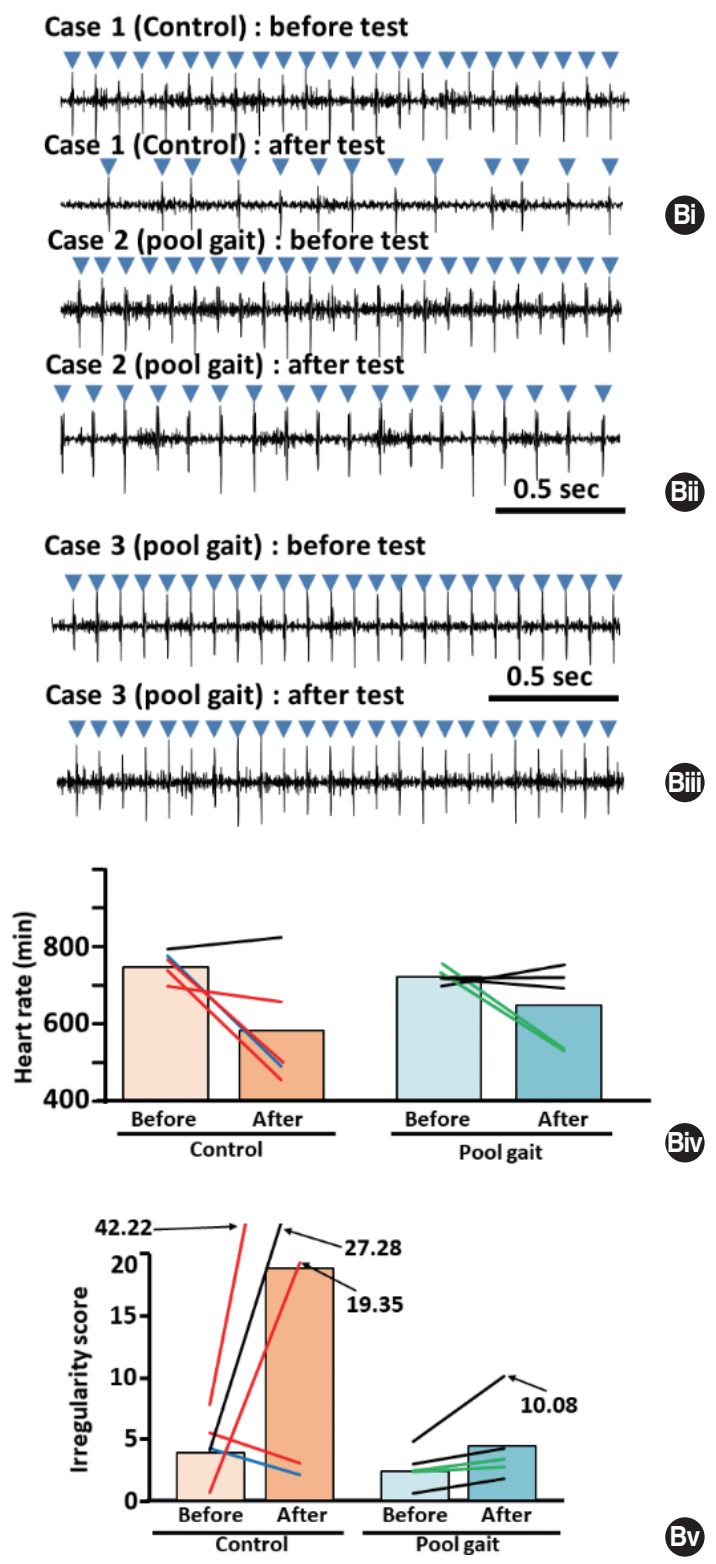

Bv 
mum speed (Fig. 7Aii and 7Aiii); however, in the control group, the value of the gait distance was more spread out than that in the pool group (black open circle; 2,302.32, blue open circle; 1,918.71, red open circles; $1,351.89,1,050.47$, and 476.06; Fig. 7Aii)

Regarding the analysis of the ECG signals between these two groups, in the control group, one in two long-distance mice (black and blue open circles) developed a decreased heart rate (Fig. 7Biv), and all three short-distance mice (red lines, Fig. 7Aii) were observed to experience suppressed heart rates (two bar graphs on the left side of Fig. 7Biv). In the pool gait group, although the heart rate in mice with high maximum speed (two green circles) decreased (two bar graphs on the right side of Fig. 7Biv), no change in heart rate was observed in mice with the low-maximum speed (two bar graphs on the left side of Fig. 7Biv). Similarly, no significant difference was observed in the average heart rate irregularity score between the groups after the gait in the water (Fig. 7Bv). In the control group, however, a drastic increase in heart rate irregularity scores was noted in 1 in 2 mice in the high-distance mice and 2 in 3 mice in the low-distance mice (a black and two red lines: two bar graphs on the left side of Fig. 7Bv). In the pool gait group, an increase in the heart rate irregularity scores was observed (a black line: two bar graphs on the right side of Fig. 7Bv); however, except for the case which was shown by the black line, the drastic increase in the heart rate irregularity score was not observed in the pool gait group (two black and two green lines: two bar graphs on the right side of Fig. 7Bv).

\section{DISCUSSION}

In this study, the effect of gait in shallow warm water on the sensory threshold, locomotive behavior, and cardiac function of FM-induced model mice were observed. It was shown that mice in the pool gait group demonstrated no statistically significant changes in electrostimulation-induced and heat-induced sensory thresholds or locomotive behavior. However, the onset time of movement after the gait in the pool gait group was significantly shorter than that in the control group, suggesting that fast motor recovery in the pool gait group may be related to cardiovascular function.

\section{Mechanisms of pool gait-induced motor recovery}

It appears that the gait in shallow warm water provided adaptability for the pool gait group because the mice were able to walk in the pool over the 3-week study period. This adaptability may have resulted from improvements in peripheral fatigue (Srikuea et al., 2013), muscle strength (Srikuea et al., 2013), lung-related maximum oxygen uptake (Andrade et al., 2017), central fatigue (Meeus et al., 2013; Schwenkreis et al., 2011), and the cardiac system (Bardal et al., 2015). Although the gait in shallow waterinduced motor recovery after the exercise seemed to relate to cardiac function (Fig. 6), we do not think that it is the only factor responsible for motor recovery. Presumably, though other factors, such as muscle strength, lung-related maximum oxygen uptake, peripheral fatigue, and central fatigue, may also accompany the cardiac system, demonstrating relationships between motor recovery and these factors was beyond the scope of this research.

\section{Mechanisms due to which pool gait-induced motor recovery did not accompany improvements in the sensory threshold}

A question arises as to why pool gait-induced motor recovery does not result in any change in sensory threshold (Fig. 4). In order to explain this, we considered the following reasons. A previous study reported that gait exercise enhances the brain's serotonin level (Pietrelli et al., 2018; Struder and Weicker, 2001a, 2001b), which upregulates the mental state (Pietrelli et al., 2018). Generally, serotonin is considered to be present in the raphe nuclei (Hornung, 2003). In addition to serotonin receptors in the brain (Hornung, 2003), serotonin also binds to receptors in the spinal dorsal horn (Tao et al., 2019; Yoshimura and Furue, 2017), which acts to inhibit pain as one of the descending inhibitory pathways (Tao et al., 2019; Yoshimura and Furue, 2017). However, the results showed no effect following exercise induction in terms of sensory threshold and locomotive behavior in this study. Therefore, we considered three serotonin-related reasons for motor recovery without any change in the sensory threshold. First, owing to the possibility of monoamine depletion in the brain, exercise-induced serotonin release might not be fully facilitated in FM-induced model mice (Nagakura et al., 2009; Oe et al., 2010). Second, serotonergic neurons may be activated, and serotonin release might be enhanced in the brain (Otsuka et al., 2016; Yoshitake et al., 2004); however, the serotonin release may have involved a temporary rather than a continuous elevation after exercise. Third, pain inhibition through training might be diminished due to exercise-induced pain enhancement (Smith et al., 2020; Staud et al., 2005). Fourth, we considered the evaluation of pain in this study. Patients with pain symptoms are generally thought to experience different types of pain, such as spontaneous pain (Nagakura et al., 2019; Tanei et al., 2020), pain during joint motion (Avila et al., 2014, 2017), pain on load (Collado-Mateo et al., 2016), and nox- 
ious stimulus-evoked pain. The pain experienced by FM patients may comprise all the pain types mentioned above. In relation to the sensory threshold, our results may only reflect the noxious stimulus-evoked pain, and other types of pain, such as motion pain and pain on load, might be diminished by the pool exercise, which may relate to motor recovery after the test.

\section{Study limitations and clinical application}

This study had some limitations. The findings showed that free gait in a shallow pool accelerated motor recovery for the pool gait group and that this recovery could be due to improved cardiac function (Fig. 7). As explained previously, the gait in shallow water-induced motor recovery cannot be attributed to improvements in cardiac function alone. However, the contribution of other factors, such as, peripheral fatigue, muscle endurance, and maximum oxygen consumption were not investigated in this study. Therefore, further studies are needed to analyze the determinants of motor recovery. Another limitation involved the location of the sensory threshold probe. The main symptom of FM is widespread musculoskeletal pain (Bazzichi et al., 2020). This study examined sensory thresholds on the plantar surface of the right hind foot. Given the fact that muscle fibers exist within the plantar surface, a simple contact between the probe and the plantar surface is an advantage in the examination of the sensory threshold on the plantar surface of the right hind foot. In this study, although sensory threshold tests were performed on the plantar surface, other muscle areas might provide an alternative probe site for sensory threshold tests, and might have led to change the results. Further, given this outcome, the method of measurement used in the determination of the sensory threshold (as "pain") might have been inappropriate in an evaluation of motion pain and pain on load. The extent of motion pain and pain on load might have been better determined by separating responses concerning locomotive behavior from motor recovery after the tests. However, methods of evaluation for the aforementioned types of pain will need to be considered in further studies.

It is important to establish therapeutic exercise programs while taking into consideration exercise ways, frequency, and intensity, for each FM patient. However, each patient has different conditions, and the intensity of widespread pain occasionally tends to be extremely low. Further, although resistance training for FM patients has already been reported (Busch et al., 2013; Figueroa et al., 2008; Glasgow et al., 2017; Kingsley et al., 2010, 2011; Valkeinen et al., 2006), the effect of high-intensity gait training on FM symptoms remains unclear. In this study, at least, the pool gait as "high-in- tensity gait training" did not worsen the sensory threshold, suggesting that these data support the possibility of high-intensity training for FM patients. Evidently, the application depends on the condition of the FM patients (Lemmey et al., 2012; van Santen et al., 2002).

In conclusion, this study aimed to investigate the effect of gait exercise in a shallow pool for FM-induced model mice. Free gait in a shallow pool accelerated motor recovery after the exercise, unlike the sensory threshold.

\section{CONFLICT OF INTEREST}

No potential conflict of interest relevant to this article was reported.

\section{ACKNOWLEDGMENTS}

This work was supported by both Kumamoto Health Science University fellowship grant (No. 2018-C-11) and a Grant-in-Aid for Scientific Research (C), JSPS to A.D. (19K11383). An illustration of the black mouse in Fig. 1A was cited from (C) 2016 DBCLS TogoTV.

\section{REFERENCES}

Altan L, Bingöl U, Aykaç M, Koç Z, Yurtkuran M. Investigation of the effects of pool-based exercise on fibromyalgia syndrome. Rheumatology Int 2004;24:272-277.

Andrade $\mathrm{CP}$, Zamuner AR, Forti M, Franca TF, Tamburus NY, Silva E. Oxygen uptake and body composition after aquatic physical training in women with fibromyalgia: a randomized controlled trial. Eur J Phys Rehabil Med 2017;53:751-758.

Avila MA, Camargo PR, Ribeiro IL, Alburquerque-Sendin F, Zamuner AR, Salvini TF. Effects of a 16-week hydrotherapy program on threedimensional scapular motion and pain of women with fibromyalgia: A single-arm study. Clin Biomech (Bristol, Avon) 2017;49:145-154.

Avila MA, Camargo PR, Ribeiro IL, Zamuner AR, Salvini TF. Three-dimensional scapular motion during arm elevation is altered in women with fibromyalgia. Clin Biomech (Bristol, Avon) 2014;29:815-821.

Bardal EM, Roeleveld K, Mork PJ. Aerobic and cardiovascular autonomic adaptations to moderate intensity endurance exercise in patients with fibromyalgia. J Rehabil Med 2015;47:639-646.

Barthe JY, Clarac F. Modulation of the spinal network for locomotion by substance P in the neonatal rat. Exp Brain Res 1997;115:485-492.

Bazzichi L, Giacomelli C, Consensi A, Giorgi V, Batticciotto A, Di Franco 
M, Sarzi-Puttini P. One year in review 2020: fibromyalgia. Clin Exp Rheumatol 2020;38 Suppl 1:3-8.

Bilberg A, Ahlmen M, Mannerkorpi K. Moderately intensive exercise in a temperate pool for patients with rheumatoid arthritis: a randomized controlled study. Rheumatology (Oxford) 2005;44:502-508.

Busch AJ, Webber SC, Richards RS, Bidonde J, Schachter CL, Schafer LA, Danyliw A, Sawant A, Dal Bello-Haas V, Rader T, Overend TJ. Resistance exercise training for fibromyalgia. Cochrane Database Syst Rev 2013;2013:CD010884

Collado-Mateo D, Adsuar JC, Olivares PR, Dominguez-Munoz FJ, Maestre-Cascales C, Gusi N. Performance of women with fibromyalgia in walking up stairs while carrying a load. PeerJ 2016;4:e1656.

da Cunha Ribeiro RP, Roschel H, Artioli GG, Dassouki T, Perandini LA, Calich AL, de Sa Pinto AL, Lima FR, Bonfa E, Gualano B. Cardiac autonomic impairment and chronotropic incompetence in fibromyalgia. Arthritis Res Ther 2011;13:R190.

Dias JM, Cisneros L, Dias R, Fritsch C, Gomes W, Pereira L, Santos ML, Ferreira PH. Hydrotherapy improves pain and function in older women with knee osteoarthritis: a randomized controlled trial. Braz J Phys Ther 2017;21:449-456.

Doi A, Miyamoto K, Nakano YS, Sakasaki J, Kasae S, Nishimura K, Shin MC, Yoshimura M. Sole vibration improves locomotion through the recovery of joint movements in a mouse cast model. PLoS One 2017; 12:e0186189.

Doi A, Sakasaki J, Tokunaga C, Sugita F, Kasae S, Nishimura K, Sato Y, Kuratsu T, Hashiguchi S, Shin MC, Yoshimura M. Both ipsilateral and contralateral localized vibratory stimulations modulated pain-related sensory thresholds on the foot in mice and humans. J Pain Res 2018; 11:1645-1657.

Figueroa A, Kingsley JD, McMillan V, Panton LB. Resistance exercise training improves heart rate variability in women with fibromyalgia. Clin Physiol Funct Imaging 2008;28:49-54.

Glasgow A, Stone TM, Kingsley JD. Resistance exercise training on disease impact, pain catastrophizing and autonomic modulation in women with fibromyalgia. Int J Exerc Sci 2017;10:1184-1195.

Hornung JP. The human raphe nuclei and the serotonergic system. J Chem Neuroanat 2003;26:331-343.

Kanda Y. Investigation of the freely available easy-to-use software 'EZR' for medical statistics. Bone Marrow Transplant. 2013;48:452-458.

Kingsley JD, McMillan V, Figueroa A. Resistance exercise training does not affect postexercise hypotension and wave reflection in women with fibromyalgia. Appl Physiol Nutr Metab 2011;36:254-263.

Kingsley JD, McMillan V, Figueroa A. The effects of 12 weeks of resistance exercise training on disease severity and autonomic modulation at rest and after acute leg resistance exercise in women with fibromyalgia.
Arch Phys Med Rehabil 2010;91:1551-1557.

Lemmey AB, Williams SL, Marcora SM, Jones J, Maddison PJ. Are the benefits of a high-intensity progressive resistance training program sustained in rheumatoid arthritis patients? A 3-year followup study. Arthritis Care Res (Hoboken) 2012;64:71-75.

Maejima $\mathrm{H}$, Inoue $\mathrm{T}$, Takamatsu Y. Therapeutic exercise accompanied by neuronal modulation to enhance neurotrophic factors in the brain with central nervous system disorders. Phys Ther Res 2019;22:38-43.

Mannerkorpi K, Nordeman L, Ericsson A, Arndorw M; GAU Study Group. Pool exercise for patients with fibromyalgia or chronic widespread pain: a randomized controlled trial and subgroup analyses. J Rehabil Med 2009;41:751-760.

Meeus M, Nijs J, Hermans L, Goubert D, Calders P. The role of mitochondrial dysfunctions due to oxidative and nitrosative stress in the chronic pain or chronic fatigue syndromes and fibromyalgia patients: peripheral and central mechanisms as therapeutic targets? Expert Opin Ther Targets 2013;17:1081-1089.

Nagakura Y, Miwa M, Yoshida M, Miura R, Tanei S, Tsuji M, Takeda H. Spontaneous pain-associated facial expression and efficacy of clinically used drugs in the reserpine-induced rat model of fibromyalgia. Eur J Pharmacol 2019;864:172716.

Nagakura Y, Oe T, Aoki T, Matsuoka N. Biogenic amine depletion causes chronic muscular pain and tactile allodynia accompanied by depression: a putative animal model of fibromyalgia. Pain 2009;146:26-33.

Oe T, Tsukamoto M, Nagakura Y. Reserpine causes biphasic nociceptive sensitivity alteration in conjunction with brain biogenic amine tones in rats. Neuroscience 2010;169:1860-1871.

Otsuka T, Nishii A, Amemiya S, Kubota N, Nishijima T, Kita I. Effects of acute treadmill running at different intensities on activities of serotonin and corticotropin-releasing factor neurons, and anxiety- and depressive-like behaviors in rats. Behav Brain Res 2016;298:44-51.

Pearson MJ, Smart NA. Exercise therapy and autonomic function in heart failure patients: a systematic review and meta-analysis. Heart Fail Rev 2018;23:91-108.

Pietrelli A, Matkovic L, Vacotto M, Lopez-Costa JJ, Basso N, Brusco A. Aerobic exercise upregulates the BDNF-Serotonin systems and improves the cognitive function in rats. Neurobiol Learn Mem 2018;155:528-542.

Schwenkreis P, Voigt M, Hasenbring M, Tegenthoff M, Vorgerd M, Kley RA. Central mechanisms during fatiguing muscle exercise in muscular dystrophy and fibromyalgia syndrome: a study with transcranial magnetic stimulation. Muscle Nerve 2011;43:479-484.

Segura-Jiménez V, Carbonell-Baeza A, Aparicio VA, Samos B, Femia P, Ruiz JR, Delgado-Fernández M. A warm water pool-based exercise program decreases immediate pain in female fibromyalgia patients: uncontrolled clinical trial. Int J Sports Med 2013;34:600-605. 
Smith TTG, Barr-Gillespie AE, Klyne DM, Harris MY, Amin M, Paul RW, Cruz GE, Zhao H, Gallagher S, Barbe MF. Forced treadmill running reduces systemic inflammation yet worsens upper limb discomfort in a rat model of work-related musculoskeletal disorders. BMC Musculoskelet Disord 2020;21:57.

Srikuea R, Symons TB, Long DE, Lee JD, Shang Y, Chomentowski PJ, Yu G, Crofford LJ, Peterson CA. Association of fibromyalgia with altered skeletal muscle characteristics which may contribute to postexertional fatigue in postmenopausal women. Arthritis Rheum 2013;65:519-528.

Staud R, Robinson ME, Price DD. Isometric exercise has opposite effects on central pain mechanisms in fibromyalgia patients compared to normal controls. Pain 2005;118:176-184.

Struder HK, Weicker H. Physiology and pathophysiology of the serotonergic system and its implications on mental and physical performance. Part I. Int J Sports Med 2001a;22:467-481.

Struder HK, Weicker H. Physiology and pathophysiology of the serotonergic system and its implications on mental and physical performance. Part II. Int J Sports Med 2001b;22:482-497.

Tanei S, Miwa M, Yoshida M, Miura R, Nagakura Y. The method simulating spontaneous pain in patients with nociplastic pain using rats with fibromyalgia-like condition. MethodsX 2020;7:100826.

Tao ZY, Wang PX, Wei SQ, Traub RJ, Li JF, Cao DY. The role of descending pain modulation in chronic primary pain: potential application of drugs targeting serotonergic system. Neural Plast 2019;2019:1389296.

Tomas-Carus P, Gusi N, Häkkinen A, Häkkinen K, Leal A, Ortega-Alonso
A. Eight months of physical training in warm water improves physical and mental health in women with fibromyalgia: a randomized controlled trial. J Rehabil Med 2008;40:248-252.

Tsai PS, Fan YC, Huang CJ. Fibromyalgia is associated with coronary heart disease: a population-based cohort study. Reg Anesth Pain Med 2015; 40:37-42.

Valkeinen H, Hakkinen A, Hannonen P, Hakkinen K, Alen M. Acute heavyresistance exercise-induced pain and neuromuscular fatigue in elderly women with fibromyalgia and in healthy controls: effects of strength training. Arthritis Rheum 2006;54:1334-1339.

van Santen M, Bolwijn P, Landewe R, Verstappen F, Bakker C, Hidding A, van Der Kemp D, Houben H, van der Linden S. High or low intensity aerobic fitness training in fibromyalgia: does it matter? J Rheumatol 2002;29:582-587.

Vlietstra L, Hendrickx W, Waters DL. Exercise interventions in healthy older adults with sarcopenia: a systematic review and meta-analysis. Australas J Ageing 2018;37:169-183.

Yoshimura M, Furue H. In vivo electrophysiological analysis of mechanisms of monoaminergic pain inhibitory systems. Pain 2017;158 Suppl:S85-S91.

Yoshitake T, Wang FH, Kuteeva E, Holmberg K, Yamaguchi M, Crawley JN, Steiner R, Bartfai T, Ogren SO, Hokfelt T, Kehr J. Enhanced hippocampal noradrenaline and serotonin release in galanin-overexpressing mice after repeated forced swimming test. Proc Natl Acad Sci U S A 2004;101:354-359. 